\title{
WEAK EQUIVALENCES OF SIMPLICIAL PRESHEAVES
}

\author{
DANIEL DUGGER AND DANIEL C. ISAKSEN
}

\begin{abstract}
Weak equivalences of simplicial presheaves are usually defined in terms of sheaves of homotopy groups. We give another characterization using relative-homotopy-liftings, and develop the tools necessary to prove that this agrees with the usual definition. From our lifting criteria we are able to prove some foundational (but new) results about the local homotopy theory of simplicial presheaves.
\end{abstract}

\section{INTRODUCTION}

In developing the homotopy theory of simplicial sheaves or presheaves, the usual way to define weak equivalences is to require that a map induce isomorphisms on all sheaves of homotopy groups. This is a natural generalization of the situation for topological spaces, but the 'sheaves of homotopy groups' machinery (see Definition 6.6) can feel like a bit of a mouthful. The purpose of this paper is to unravel this definition, giving a fairly concrete characterization in terms of lifting properties - the kind of thing which feels more familiar and comfortable to the ingenuous homotopy theorist.

The original idea came to us via a passing remark of Jeff Smith's: He pointed out that a map of spaces $X \rightarrow Y$ induces an isomorphism on homotopy groups if and only if every diagram

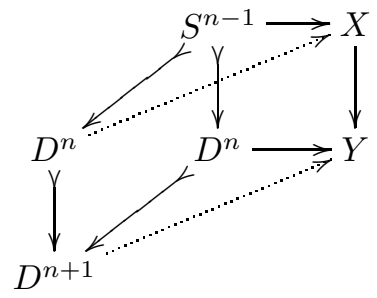

admits liftings as shown (for every $n \geq 0$, where by convention we set $S^{-1}=\emptyset$ ). Here the maps $S^{n-1} \hookrightarrow D^{n}$ are both the boundary inclusion, whereas the two maps $D^{n} \hookrightarrow D^{n+1}$ in the diagram are the two inclusions of the surface hemispheres of $D^{n+1}$. The map $D^{n+1} \rightarrow Y$ should be thought of as giving a homotopy between the two maps $D^{n} \rightarrow Y$ relative to $S^{n-1}$. In essence, the above lifting condition just guarantees the vanishing of the relative homotopy groups of $X \rightarrow Y$.

One advantage of this formulation is that one doesn't have to worry about basepoints, but it also has other conveniences. If one looks back on the classical lifting theorems in [Sp], for instance, it is really the above property - rather than the isomorphism on homotopy groups - which is being made use of over and over again. In working with simplicial presheaves, it eventually became clear that a version of 
the above characterization was a useful thing to have around. It comes in at several points in [DHI], where it is used to inductively produce liftings much like in [Sp].

Whereas for topological spaces the above characterization is 'obvious', for simplicial presheaves it requires a little bit of work. Intuitively the result is clear, but to actually write down a proof one must (a) struggle with the combinatorics of simplicial sets, and (b) deal with the 'local homotopy theory' which demands that everything be accomplished by a finite number of lifting arguments. The trouble is that the modern way of avoiding (a) is to use the tools of model categories, but because of (b) we don't have these at our disposal. In this paper we develop some basic machinery for handling this situation, so that in the end one can write out the proof fairly smoothly.

To describe the results more explicitly, we'll first give an analagous characterization for weak equivalences $X \rightarrow Y$ of simplicial sets. In this case, we must assume that $X$ and $Y$ are fibrant. The $(n-1)$-sphere is replaced by $\partial \Delta^{n}$; the $n$-disks are replaced by $\Delta^{n}$; and $D^{n+1}$ is replaced by the pushout $R H\left(\Delta^{n}, \partial \Delta^{n}\right)$ of the diagram

$$
\partial \Delta^{n} \leftarrow \partial \Delta^{n} \times \Delta^{1} \rightarrow \Delta^{n} \times \Delta^{1} .
$$

The simplicial set $R H\left(\Delta^{n}, \partial \Delta^{n}\right)$ is the domain of simplicial homotopies-relative to $\partial \Delta^{n}$ - between maps out of $\Delta^{n}$. Once these substitutions are made into diagram (1.1), one gets the same criterion for the map $X \rightarrow Y$ to be a weak equivalence of simplicial sets. See Proposition 4.1.

The generalization to the case of simplicial presheaves is now reasonably transparent. The main result of the paper is the following:

Theorem 1.2. Let $\mathcal{C}$ be a Grothendieck site, and let $F \rightarrow G$ be a map between locally fibrant simplicial presheaves. Then $F \rightarrow G$ induces an isomorphism on all sheaves of homotopy groups (for all choices of basepoint) if and only if it has the following property: for every solid-arrow diagram

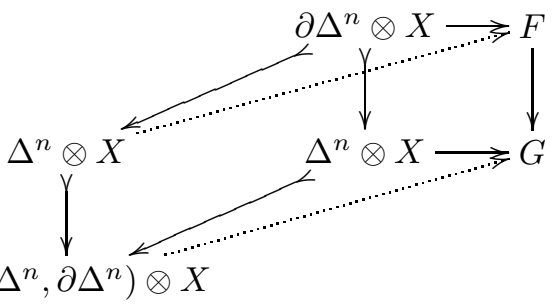

in which $X$ is representable, there exists a covering sieve of $X$ such that for any $U \rightarrow X$ in the sieve, the diagram obtained by pulling back to $U$ has liftings as shown.

(The dotted arrows in the above diagram are called 'relative-homotopy-liftings', and the fact that they only exist locally leads us to call this property the 'local RHLP'- see Defintion 6.11, as well as the general discussion in Section 3).

The advantage of this viewpoint on weak equivalences is that it provides a framework for using lifting arguments instead of computations of homotopy groups. For questions in homotopy theory, lifting is sometimes a more convenient tool. In our case, this lifting characterization will be used to give elegant proofs of various results (some old, some new) about local weak equivalences and local-fibrations. These are given in Section 7. To readers of [J1, J3], these come as no surprise. However, our 
proofs seem simpler and more conceptual than the ones involving sheaves of homotopy groups, in particular avoiding all references to stalks or Boolean localizations. And in some cases we don't know any proof other than via the lifting criterion.

We should remark that this approach via liftings is not at all meant to replace the definition involving sheaves of homotopy groups - in some situations that is exactly the tool that is needed. But in general it is good to have both descriptions at one's disposal.

The chief motivation for writing this paper was its application to our study of localization for simplicial presheaves [DHI]. But we've also found that the techniques of homotopy-liftings are convenient tools to have around, and should be more wellknown among abstract homotopy theorists. Reedy $[R]$ worked with a dual version of these lifting criterion in the context of abstract model categories, and used them to prove several key lemmas. We have reproduced a couple of his proofs here for completeness, and with the goal of popularizing these ideas.

\subsection{Organization of the paper.}

Sections $2-5$ deal only with simplicial sets. Section 2 has a few background results, and then in Section 3 we define relative-homotopy-liftings and develop their basic properties. In Section 4 these ideas are applied to get a lifting criterion for weak equivalences of simplicial sets. Unfortunately, one of the key steps is easiest to prove using model-category theoretic methods, and these do not generalize to the simplicial presheaf setting. So Section 5 is devoted to giving a completely combinatorial proof for this result. We finally get to simplicial presheaves in Section 6 . We recall the traditional definition of weak equivalence using sheaves of homotopy groups, and then prove Theorem 1.2. Section 7 concludes with some applications of this theorem.

We assume that the reader is familiar with standard results from the homotopy theory of simplicial sets, including material to be found in $[\mathrm{M}]$ or [GJ]. Much of what we discuss, especially in Sections 3 and 4, can be easily generalized to abstract model categories, but we do not treat this extra generality here. We'll also assume a familiarity with sheaf theory and the homotopy theory of simplicial presheaves, for which we refer the reader to [J1]. It should be clear from our arguments how indebted we are to that paper.

\section{BACKGROUND ON SIMPLICIAL SETS}

We start with some basic facts. Let $S^{n}$ be the sphere $\Delta^{n} / \partial \Delta^{n}$. If $(K, x)$ is a pointed simplicial set, then $\pi_{n}(K, x)$ denotes the set of maps $\left(\Delta^{n}, \partial \Delta^{n}\right) \rightarrow(K, x)$ modulo the equivalence relation generated by simplicial homotopy relative to $\partial \Delta^{n}$. Of course this set has homotopical meaning only if $K$ is fibrant.

In $[\mathrm{K}]$, Kan constructed a fibrant-replacement functor called $\mathrm{Ex}^{\infty}$. First, let sd be the barycentric subdivision functor [GJ, p. 183]. For any simplicial set $X$, $\operatorname{Ex} X$ is the simplicial set whose $k$-simplices are elements of the set $\operatorname{Hom}\left(\operatorname{sd} \Delta^{k}, X\right)$. The functor Ex is right adjoint to sd. Now $\mathrm{Ex}^{n}$ is the $n$-fold composition of Ex, and $\mathrm{Ex}^{\infty}$ is $\operatorname{colim}_{n} \mathrm{Ex}^{n}$. The functor $\mathrm{Ex}^{\infty}$ has some nice properties one wouldn't expect from an arbitrary fibrant-replacement functor: It preserves fibre-products, it preserves the set of 0 -simplices, and it preserves fibrations. These properties all follow immediately from the definition.

The following two basic lemmas about simplicial sets will be used later. The first is obvious, but its statement and proof becomes important when considering 
simplicial presheaves later. The point is that the proof uses only basic lifting properties, not fancy model theoretic results.

Lemma 2.1. Let $i: K \hookrightarrow L$ be a cofibration, and let $X$ be a fibrant simplicial set. If $f: K \rightarrow X$ factors through any contractible simplicial set $M$, then $f$ is simplicially null-homotopic and $f$ extends over $i$.

Proof. For any simplicial set $Y$, let Cone $Y$ be $\left(Y \times \Delta^{1}\right) /(Y \times\{1\})$. We have a diagram

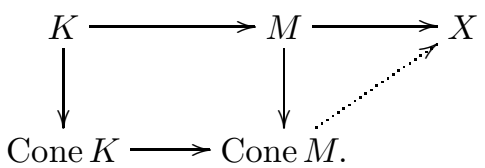

The map $M \rightarrow$ Cone $M$ is an acyclic cofibration because $M$ is contractible, so there is a lift as shown. Composition with Cone $K \rightarrow$ Cone $M$ gives the desired simplicial null-homotopy.

Now we have a diagram

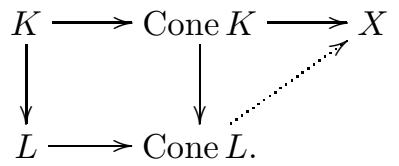

The map Cone $K \rightarrow$ Cone $L$ is an acyclic cofibration, so there is a lift as shown. Composition with $L \rightarrow$ Cone $L$ gives the desired extension over $i$.

Note that if $K, L$, and $M$ are finite simplicial sets, then the desired lift can be produced using only finitely many applications of the Kan extension condition for $X$. This will be important when we start generalizing to simplicial presheaves.

Lemma 2.2. Let $i: K \rightarrow L$ be an acyclic cofibration between finite simplicial sets. Then $i$ can be built from the maps $\Lambda^{n, k} \hookrightarrow \Delta^{n}$ by a finite number of retracts, cobase changes, and compositions.

Proof. We know that $i$ is a retract of a relative $J$-cell complex $j: M \rightarrow N$ (cf. [H, Def. 12.5.8]), where $J$ is the set of maps of the form $\Lambda^{n, k} \hookrightarrow \Delta^{n}$. Since $L$ is finite, its image in $N$ belongs to a finite subcomplex. Thus $i$ is actually a retract of a finite relative $J$-cell complex.

\section{Generalities about homotopy-Liftings}

This section establishes the definition and basic properties of what we call 'relative-homotopy-liftings'.

Definition 3.1. A square of simplicial sets

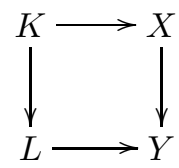

is said to have a relative-homotopy-lifting if there exists a map $L \rightarrow X$ such that the upper left triangle commutes and there is a simplicial homotopy relative to $K$ from the composition $L \rightarrow X \rightarrow Y$ to the given map $L \rightarrow Y$.

The map $X \rightarrow Y$ has the relative-homotopy-lifting property (RHLP) with respect to $K \rightarrow L$ if every square (3.2) has a relative-homotopy-lifting. 
Reedy [R, Lem. 2.1] used the dual to the above definition. Like him, we could haved defined this property in an arbitrary simplicial model category (one probably doesn't even need the model category to be simplicial). All of our basic results go through in that generality, but we won't ever need this.

Given $K \rightarrow L$, let $\boldsymbol{R H}(\boldsymbol{L}, \boldsymbol{K})$ denote the pushout of the diagram

$$
K \stackrel{\pi}{\longleftarrow} K \times \Delta^{1} \longrightarrow L \times \Delta^{1}
$$

where the left map is the projection. The notation stands for 'Relative-Homotopy': To give a map $R H(L, K) \rightarrow X$ means precisely to give two maps $L \rightarrow X$ which agree on $K$, together with a simplicial homotopy between them relative to $K$. Note that there is a canonical map $L \amalg_{K} L \rightarrow R H(L, K)$. This map is a cobase change of the map

$$
\left(K \times \Delta^{1}\right) \cup\left(L \times \partial \Delta^{1}\right) \rightarrow L \times \Delta^{1},
$$

so it is a cofibration if $K \rightarrow L$ is.

We will sometimes use the fact that the existence of relative-homotopy-liftings can be rephrased as saying that the diagram

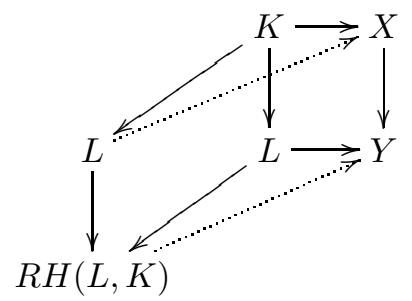

admits liftings as shown. While this diagram may seem somewhat awkward (especially when seeing it for the first time), it is often a very useful tool.

Here are some basic properties of relative-homotopy-liftings:

Lemma 3.4. Let $f: X \rightarrow Y$ be a fixed map of simplicial sets. Consider the class of all maps $K \rightarrow L$ with respect to which $f$ has the RHLP. This class is closed under cobase changes and retracts. If $Y$ is fibrant, then the composition of two cofibrations in the class is still in the class.

Proof. Closure under cobase changes follows from consideration of (3.3) and the fact that $R H\left(L \amalg_{K} M, M\right)$ is isomorphic to $R H(L, K) \amalg_{K} M$. Closure under retracts follows from the usual formal argument with lifting properties.

For composition, we start with two cofibrations $i: K \rightarrow L$ and $j: L \rightarrow M$ in the class. Consider a lifting problem

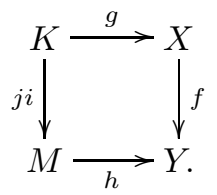

The first step is to produce a homotopy-lifting

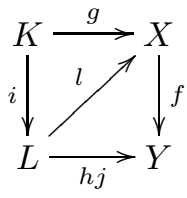


relative to $K$. Let $H: R H(L, K) \rightarrow Y$ be the relative-homotopy from $f l$ to $h j$. So now we look at the diagram

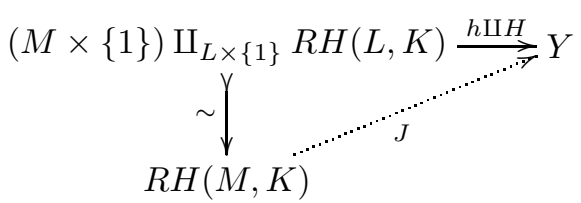

and produce a lifting $J$ using that $Y$ is fibrant. Note that the vertical map above is an acyclic cofibration because it is a cobase change of the acyclic cofibration

$$
(M \times\{1\}) \cup\left(L \times \Delta^{1}\right) \rightarrow M \times \Delta^{1} .
$$

Let $m$ be the map $\left.J\right|_{M \times\{0\}}$. Note that $m$ is simplicially homotopic to $h$ relative to $K$.

At this point we have the square

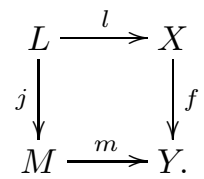

We produce a relative-homotopy-lifting $n$. A diagram chase shows that $n j i$ equals $g$. On the other hand, $f n$ is simplicially homotopic to $m$ relative to $K$ and hence also to $h$; here we use that relative-homotopy is transitive because $Y$ is fibrant.

Corollary 3.5. If $f: X \rightarrow Y$ has the RHLP with respect to the maps $\partial \Delta^{n} \hookrightarrow$ $\Delta^{n}$ for all $n \geq 0$ and $Y$ is fibrant, then $p$ also has the RHLP with respect to all cofibrations $K \hookrightarrow L$ of finite simplicial sets.

Proof. Every such cofibration $K \hookrightarrow L$ can be constructed by a finite number of compositions and cobase changes from the generating cofibrations $\partial \Delta^{n} \hookrightarrow \Delta^{n}$.

Proposition 3.6. Suppose that $K \hookrightarrow L$ is a cofibration and we are given a square $\mathcal{S}$ of the form

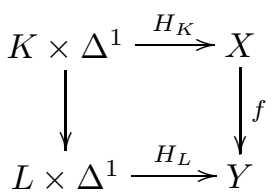

in which $X$ and $Y$ are fibrant. Let $\mathcal{S}_{0}$ denote the square obtained by restricting $H_{K}$ and $H_{L}$ to time $t=0$, and similarly for $\mathcal{S}_{1}$. Then $\mathcal{S}_{0}$ has a relative-homotopy-lifting if and only if $\mathcal{S}_{1}$ does.

Proof. Suppose that $S_{0}$ has a relative-homotopy-lifting $l_{0}$. Then by gluing $l_{0}$ to $H_{K}$ we get $(L \times\{0\}) \cup\left(K \times \Delta^{1}\right) \rightarrow X$, and since $X$ is fibrant this map extends over $L \times \Delta^{1}$. Let $l_{1}$ denote the restriction of this map to $L \times\{1\}$; we will show that $l_{1}$ is the desired relative-homotopy-lifting.

Pushing the homotopy $L \times \Delta^{1} \rightarrow X$ down into $Y$, we can glue it to $H_{L}$ to get a map defined on $\left(L \amalg_{K} L\right) \times \Delta^{1}$. Together with the relative-homotopy from $f l_{0}$ to $\left.H_{L}\right|_{t=0}$, we find that we actually have a map $[R H(L, K) \times\{0\}] \cup\left[\left(L \amalg_{K} L\right) \times \Delta^{1}\right] \rightarrow Y$. Since $Y$ is fibrant, this extends over $R H(L, K) \times \Delta^{1}$. Restricting to time $t=1$ gives the desired relative-homotopy of $f l_{1}$ with $\left.H_{L}\right|_{t=1}$. 
Proposition 3.7. Let $f: X \rightarrow Y$ be a map between fibrant simplicial sets. Then $f$ has the RHLP with respect to every acyclic cofibration $K \hookrightarrow L$. In particular, $f$ has the RHLP with respect to the maps $\Lambda^{n, k} \hookrightarrow \Delta^{n}$.

Proof. Consider a square

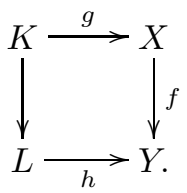

Using that $X$ is fibrant, there is a map $l: L \rightarrow X$ extending $K \rightarrow X$. We must give a relative-homotopy from $f l$ to $h$.

The cofibration $L \amalg_{K} L \hookrightarrow R H(L, K)$ is an acyclic cofibration because it is a cobase change of the acyclic cofibration $\left(L \times \partial \Delta^{1}\right) \cup\left(K \times \Delta^{1}\right) \hookrightarrow L \times \Delta^{1}$. We may extend the map $f l \amalg h: L \amalg_{K} L \rightarrow Y$ to $R H(L, K) \rightarrow Y$ because $Y$ is fibrant. This gives us the desired relative-homotopy.

Observe that if $K \rightarrow L$ is $\Lambda^{n, k} \rightarrow \Delta^{n}$ then the proof only requires a finite number of uses of the Kan extension condition. Combined with Lemma 2.2, this tells us that the proposition applies to the simplicial presheaf setting whenever $K$ and $L$ are finite simplicial sets. This won't be needed until Section 6 .

\section{WEAK EQUiVALENCES OF SIMPLICIAL SETS}

The following proposition now shows that weak equivalences between fibrant simplicial sets can be detected using relative-homotopy-liftings. This is analogous to the situation discussed in the introduction for topological spaces, where every object is fibrant.

Proposition 4.1. A map $f: X \rightarrow Y$ between fibrant simplicial sets is a weak equivalence if and only if it has the RHLP with respect to the maps $\partial \Delta^{n} \hookrightarrow \Delta^{n}$, for all $n \geq 0$.

When the simplicial sets are not fibrant one has to allow oneself to subdivide $\partial \Delta^{n}$ and $\Delta^{n}$, but we won't pursue this.

The following proof is similar to the proof of [R, Lem. 2.1]. The difference is that we only consider the RHLP with respect to the generating cofibrations, while Reedy considers the RHLP with respect to all cofibrations. We include the full details for completeness.

Proof. First suppose that $f$ has the RHLP. By Corollary 3.5, $f$ has the RHLP with respect to the cofibrations $* \hookrightarrow S^{n}$ for all $n \geq 1$, as well as $\emptyset \rightarrow *$. This shows that $\pi_{n} X \rightarrow \pi_{n} Y$ is surjective (for any choice of basepoint). Similarly, $f$ has the RHLP with respect to the cofibrations $S^{n} \vee S^{n} \hookrightarrow R H\left(S^{n}, *\right)$ for all $n$. This shows that $\pi_{n} X \rightarrow \pi_{n} Y$ is injective.

Conversely, we'll now suppose that $f$ is a weak equivalence. Consider a square

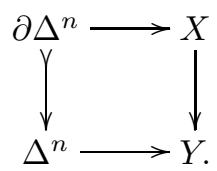


Factor $f$ into an acyclic cofibration $i: X \hookrightarrow Z$ followed by an acyclic fibration $p: Z \rightarrow Y$. Since $X$ is fibrant, there is a map $g: Z \rightarrow X$ making $X$ a retract of $Z$. Now the square

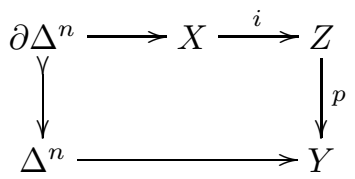

has a lift $h$ because $p$ is an acyclic fibration.

The composition $g h$ is the desired homotopy-lift. Using that $g i$ is the identity, the upper left triangle commutes. Working in the undercategory $\partial \Delta^{n} \downarrow s \mathcal{S} e t$, we see that $i g$ represents the same map as $\mathrm{id}_{Z}$ in the homotopy category - therefore pigh represents the same map as $p h$ in the homotopy category. But these latter two maps have cofibrant domain and fibrant target, so they are actually simplicially homotopic in $\partial \Delta^{n} \downarrow s$ Set. The simplicial set $R H\left(\Delta^{n}, \partial \Delta^{n}\right)$ is precisely a cylinder object for $\Delta^{n}$ in this undercategory, so pigh and $p h$ are simplicially homotopic relative to $\partial \Delta^{n}$.

Reedy $[\mathrm{R}, \mathrm{Th} . \mathrm{B}]$ showed that base changes along fibrations preserve weak equivalences between fibrant objects. His proof used the criterion of Proposition 4.1 (suitably generalized to arbitrary model categories) to detect weak equivalences. We use this idea to obtain the following elementary proof of right properness for simplicial sets - most standard references [GJ, H] use topological spaces to prove this. We include full details because this same proof will be applied to the case of simplicial presheaves.

Corollary 4.2 (Right properness). Let $f: X \rightarrow Y$ be a weak equivalence of simplicial sets, and let $p: Z \rightarrow Y$ be a fibration. Then the map $X \times_{Y} Z \rightarrow Z$ is also a weak equivalence.

Proof. We need only show that $\operatorname{Ex}^{\infty}\left(X \times_{Y} Z\right) \rightarrow \operatorname{Ex}^{\infty} Z$ is a weak equivalence. Note that $\mathrm{Ex}^{\infty}$ commutes with fibre-products and preserves fibrations, so this map is a base change of the weak equivalence $\operatorname{Ex}^{\infty} f$ along the fibration $\operatorname{Ex}^{\infty} p$. Therefore, we may assume that $X, Y$ and $Z$ are already fibrant.

The rest of the proof is the same as Reedy's argument. Suppose given a square

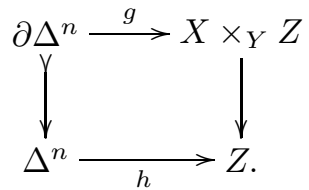

We want to find a relative-homotopy-lifting for this square. First, take a relativehomotopy-lifting $l$ for the composite square

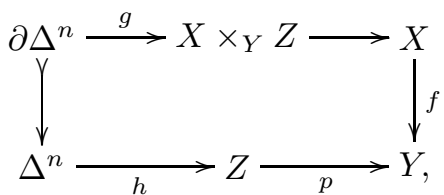


which exists by Proposition 4.1 because $X \rightarrow Y$ is a weak equivalence between fibrant simplicial sets. Now consider the square

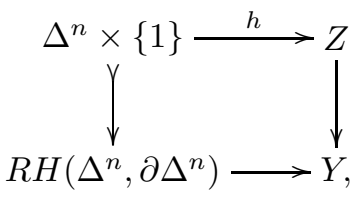

where the bottom horizontal map is the relative-homotopy from $f l$ to $p h$. This square has a lift $H$ because the left vertical arrow is an acyclic cofibration. Let $H_{0}$ and $H_{1}$ be the restrictions of $H$ to $\Delta^{n} \times\{0\}$ and $\Delta^{n} \times\{1\}$ respectively. Note that $H_{1}=h$ and $p H_{0}=f l$.

The maps $l$ and $H_{0}$ together define a map $m: \Delta^{n} \rightarrow X \times_{Y} Z$. A diagram chase shows that $g$ is the restriction of $m$ to $\partial \Delta^{n}$, and $H$ is the necessary relativehomotopy.

\section{A COMbinatorial PRoof}

In the previous section, Proposition 4.1 compared weak equivalences between fibrant simplicial sets to maps that have the RHLP with respect to the cofibrations $\partial \Delta^{n} \hookrightarrow \Delta^{n}$. Unfortunately, the proof of one implication of the proposition relied on model category theoretic methods. When we generalize to simplicial presheaves later on, these methods are not at our disposal. Thus, our goal in this section is to show by purely combinatorial methods that if $f$ is a weak equivalence then it has the RHLP with respect to the maps $\partial \Delta^{n} \hookrightarrow \Delta^{n}$.

Throughout this section $f: X \rightarrow Y$ denotes a map between fibrant simplicial sets.

First note that surjectivity on homotopy groups says precisely that $f$ has the RHLP with respect to the maps $* \rightarrow S^{n}$ for all $n \geq 1$ as well as the map $\emptyset \rightarrow *$. Using this, we have:

Lemma 5.1. If $f: X \rightarrow Y$ is a map between fibrant simplicial sets that induces surjections on homotopy groups, then $f$ has the RHLP with respect to the maps $\Lambda^{n, k} \hookrightarrow \partial \Delta^{n}$, for any $n \geq 1$.

Proof. Suppose given a square

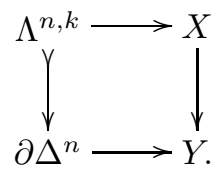

Since $\Lambda^{n, k}$ is contractible and $X$ is fibrant, the map $\Lambda^{n, k} \rightarrow X$ is simplicially nullhomotopic (by Lemma 2.1) - choose a null-homotopy. By composing with $X \rightarrow Y$, we also get a null-homotopy for the composition $\Lambda^{n, k} \rightarrow Y$; so we have a map $\left(\partial \Delta^{n} \times\{0\}\right) \cup\left(\Lambda^{n, k} \times \Delta^{1}\right) \rightarrow Y$ that is constant on $\Lambda^{n, k} \times\{1\}$. Because $Y$ is fibrant, this map extends to a map $\partial \Delta^{n} \times \Delta^{1} \rightarrow Y$ that is constant on $\Lambda^{n, k} \times\{1\}$. 
We have constructed a homotopy (in the sense of Proposition 3.6) between the original square and a square of the form

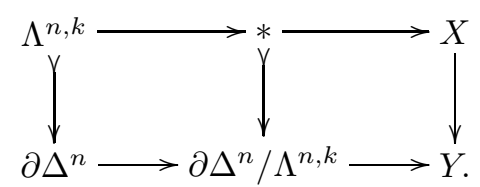

By Proposition 3.6, we need only construct a relative-homotopy-lifting for this new square. The left square is a pushout, so we need only construct a relative-homotopylifting for the right-hand square by Lemma 3.4.

Note that $\partial \Delta^{n} / \Lambda^{n, k}$ is isomorphic to $S^{n-1}$. Therefore, $f$ has the RHLP with respect to $* \rightarrow \partial \Delta^{n} / \Lambda^{n, k}$ because $f$ induces a surjection on $(n-1)$ st homotopy groups.

Theorem 5.2. If $f: X \rightarrow Y$ is a weak equivalence between fibrant simplicial sets, then it has the RHLP with respect to the maps $\partial \Delta^{n} \hookrightarrow \Delta^{n}$, for all $n \geq 0$.

Proof. Surjectivity on $\pi_{0}$ immediately gives the result for $n=0$. So suppose $n \geq 1$ and we have a lifting diagram

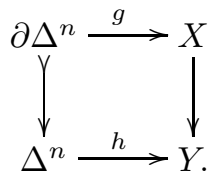

Routine lifting arguments show that there is a simplicial homotopy $\partial \Delta^{n} \times \Delta^{1} \rightarrow X$ between $g$ and a map that factors through $\partial \Delta^{n} / \Lambda^{n, n}$. As in the proof of Lemma 5.1 , we can extend this to a simplicial homotopy $\Delta^{n} \times \Delta^{1} \rightarrow Y$, and we are reduced to producing a relative-homotopy-lifting for a square of the form

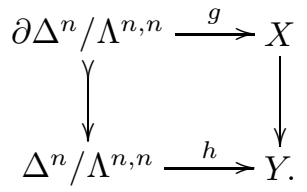

Note that $\partial \Delta^{n} / \Lambda^{n, n}$ is isomorphic to $S^{n-1}$ and $\Delta^{n} / \Lambda^{n, n}$ is contractible. Lemma 2.1 shows that $g: S^{n-1} \rightarrow X$ becomes null in $\pi_{n-1}(Y)$. Since $f$ is injective on homotopy groups, $g$ is simplicially null-homotopic. Therefore, $g$ extends to a map $l: \Delta^{n} / \Lambda^{n, n} \rightarrow X$ by Lemma 2.1 .

Define a map $H: \partial \Delta^{n+1} \rightarrow Y$ by making the $(n+1)$ st face equal to $h$, the $n$th face equal to $f l$, and all the other faces equal to the basepoint $*$. Similarly, define $J: \Lambda^{n+1, n+1} \rightarrow X$ by making the $n$th face equal to $l$ and all the other faces equal to the basepoint. So we have a square

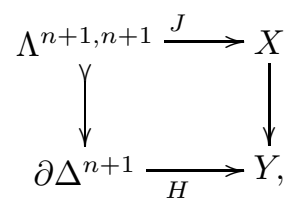

and by Lemma 5.1 this has a relative-homotopy-lifting $m$. The $(n+1)$ st face of $m$ gives a map $\Delta^{n} \rightarrow X$ that is a relative-homotopy-lifting for our original square. 


\section{Local WEAK EQUiVAlENCES OF SIMPLiCIAL PRESHEAVES}

In this section we prove the main theorem (stated here as Theorem 6.15). We start by recalling some of the tools from [J1]: the use of local lifting properties and sheaves of homotopy groups. Then we set up the local version of relativehomotopy-liftings, and observe that everything we've done so far still works in this setting.

6.1. Local-liftings. Fix a Grothendieck site $\mathcal{C}$. Recall that a map of simplicial presheaves $F \rightarrow G$ is a local-fibration if it has the following property: given any square

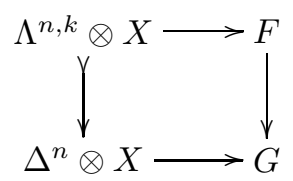

in which $X$ is representable, there exists a covering sieve of $X$ such that for any map $U \rightarrow X$ in the sieve, the induced diagram

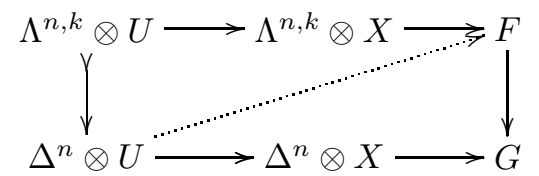

has a lifting as shown. We are not requiring that the liftings for different $U$ 's be compatible in any way, only that they exist. This kind of 'local lifting property' will appear often in the course of the paper, so we adopt the following convention:

Convention 6.4. Suppose given a lifting diagram like (6.2), in which a representable presheaf $X$ appears. We say this diagram has local liftings if there exists a covering sieve $R$ of $X$ such that for any $U \rightarrow X$ in $R$ the diagram obtained by pulling back to $U$ admits liftings. For instance, using this language, a map $F \rightarrow G$ is a local-fibration provided that every diagram (6.2) admits local liftings.

Because of Lemma 2.2, a map is a local-fibration if and only if it has the local right lifting property with respect to all maps $K \otimes X \hookrightarrow L \otimes X$ for every acyclic cofibration $K \hookrightarrow L$ between finite simplicial sets.

6.5. Sheaves of homotopy groups. Let $F$ be a simplicial presheaf on $\mathcal{C}$. Given an object $X$ of $\mathcal{C}$ and a 0 -simplex $x$ in $F(X)$, we define presheaves $\pi_{n}(F, x)$ on the site $\mathcal{C} \downarrow X$ by the formula $U \mapsto \pi_{n}\left(F(U),\left.x\right|_{U}\right)$.

Definition 6.6. A map of simplicial presheaves $f: F \rightarrow G$ is a local weak equivalence if

(1) The induced map $\pi_{0} F \rightarrow \pi_{0} G$ yields an isomorphism upon sheafification, and

(2) For every $X$ in $\mathcal{C}$ and every basepoint $x$ in $F_{0}(X)$, the map of presheaves on $\mathcal{\downarrow} \downarrow X$ given by $\pi_{n}\left(\mathrm{Ex}^{\infty} F, x\right) \rightarrow \pi_{n}\left(\mathrm{Ex}^{\infty} G, f x\right)$ also becomes an isomorphism upon sheafification. (Here $\operatorname{Ex}^{\infty} F$ is the presheaf $U \mapsto \mathrm{Ex}^{\infty}(F(U)$ ), of course).

Local weak equivalences are called 'topological weak equivalences' in [J1]. One can also use the presheaf $\pi_{n}^{l o c}(F, x)$, whose value on an object $U \rightarrow X$ is the set of based maps $S^{n} \rightarrow F(U)$ modulo the equivalence relation generated by local simplicial homotopy (see [J1, p. 44]). Two maps $S^{n} \rightarrow F(U)$ are locally simplicially homotopic if there exists a covering sieve of $U$ such that for every $V \rightarrow U$ in 
the sieve, the two restrictions $S^{n} \rightarrow F(V)$ are simplicially homotopic as based maps. The following result appears in [J1, Prop. 1.18], except for an unnecessary hypothesis.

Lemma 6.7. The map $\pi_{n}(F, x) \rightarrow \pi_{n}^{l o c}(F, x)$ is an isomorphism after sheafification, for any simplicial presheaf $F$.

Before proving Lemma 6.7, we recall the following property of sheafifications.

Lemma 6.8. A map $f: F \rightarrow G$ between presheaves of sets induces an isomorphism on sheafifications if and only if the following two conditions are satisfied:

(1) Given any $X$ in $\mathcal{C}$ and any $s$ in $G(X)$, there is a covering sieve $R$ of $X$ such that the restriction $\left.s\right|_{U}$ belongs to the image of $F(U)$ in $G(U)$ for any element $U \rightarrow X$ of $R$;

(2) Given any $X$ in $\mathcal{C}$ and any two sections $s$ and $t$ in $F(X)$ such that $f(s)=f(t)$, there exists a covering sieve $R$ of $X$ such that $\left.s\right|_{U}=\left.t\right|_{U}$ in $F(U)$ for every element $U \rightarrow X$ of $R$.

Proof. Condition (2) is equivalent to $F^{+} \rightarrow G^{+}$being an objectwise monomorphism, which in turn is equivalent to the same property for $F^{++} \rightarrow G^{++}$. If $F^{++} \rightarrow G^{++}$is an objectwise surjection then property (1) is easily seen to hold. Finally, properties (1) and (2) together imply that $\operatorname{im}\left(G(X) \rightarrow G^{++}(X)\right) \subseteq$ $\operatorname{im}\left(F^{++}(X) \hookrightarrow G^{++}(X)\right)$. From this one deduces that $F^{++} \rightarrow G^{++}$is an objectwise surjection (using that the domain and codomain are sheaves).

We will make use of the above two conditions in studying sheaves of homotopy groups.

Proof of Lemma 6.7. Since local simplicial homotopy is a larger equivalence relation than simplicial homotopy, the map is an objectwise surjection. This verifies condition (1) of Lemma 6.8.

For condition (2), suppose that $s$ and $t$ are two maps $S^{n} \rightarrow F(U)$ that are related by a finite chain of local simplicially homotopies. There is a finite sequence $s=s_{0}, s_{1}, \ldots, s_{n}=t$ of maps $S^{n} \rightarrow F(U)$ such that $s_{i}$ and $s_{i+1}$ are simplicially homotopic after restricting to a sieve $R_{i}$. Taking $R$ to be a common refinement of each $R_{i}$, we conclude that $s$ and $t$ are related by a chain of simplicial homotopies after restricting to $R$. This verifies condition (2) of Lemma 6.8.

In general, $\pi_{n}^{l o c}(F, x)$ does not carry homotopical information unless $F$ is locallyfibrant. At first glance, the sheafification of $\pi_{n}(F, x)$ seems not to be homotopically meaningful unless $F$ is objectwise fibrant, but Lemma 6.7 shows that we only need $F$ to be locally-fibrant. In other words, for locally-fibrant simplicial presheaves one can ignore the presence of $\mathrm{Ex}^{\infty}$ in Definition 6.6:

Proposition 6.9. If $F$ and $G$ are locally-fibrant, then a map $f: F \rightarrow G$ is a local weak equivalence if and only if

(1) The induced map $\pi_{0} F \rightarrow \pi_{0} G$ yields an isomorphism upon sheafification, and

(2) For every $X$ in $\mathcal{C}$ and every basepoint $x$ in $F_{0}(X)$, the map $\pi_{n}(F, x) \rightarrow$ $\pi_{n}(G, f x)$ is an isomorphism upon sheafification. 
Proof. Consider the square

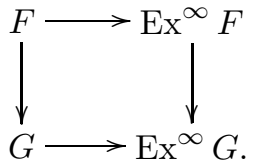

By [J1, Prop. 1.17] and Lemma 6.8, the horizontal maps satisfy the above conditions. Therefore the left vertical map satisfies the conditions if and only if the right vertical map does. The usual complications with choosing basepoints do not arise because $\mathrm{Ex}^{\infty}$ preserves 0 -simplices.

6.10. Local relative-homotopy-liftings. The relative-homotopy-lifting criterion for weak equivalences of simplicial sets (given in Proposition 4.1) has an obvious extension to the presheaf category in which we only require local liftings.

Definition 6.11. Let $K \rightarrow L$ be a map of simplicial sets. A map $f: F \rightarrow G$ of simplicial presheaves is said to have the local $\mathbf{R H L P}$ with respect to $K \rightarrow L$ if every diagram

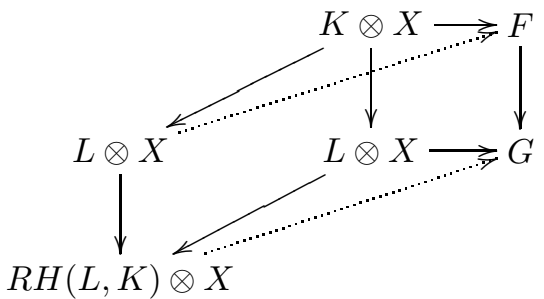

admits local liftings.

In other words, the definition requires that there exists a covering sieve of $X$ such that for any map $U \rightarrow X$ in the sieve, the induced diagram

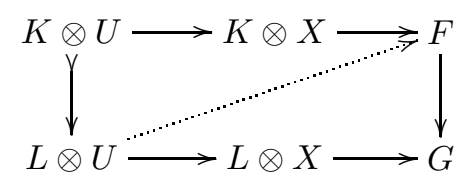

has a relative-homotopy-lifting. The liftings and simplicial homotopies one gets as $U$ varies need not be compatible in any way.

The basic results about relative-homotopy-liftings from Section 3 all go through in the present context. One only has to observe that the arguments require finitely many uses of the lifting conditions. The following result will be especially useful to us:

Lemma 6.13. Let $f: F \rightarrow G$ be a fixed map of simplicial presheaves. Consider the class of all maps $K \rightarrow L$ of simplicial sets with respect to which $f$ has the local RHLP. This class is closed under cobase changes and retracts. If $G$ is locally-fibrant, then the cofibrations in this class are also closed under composition.

Proof. The proof is the same as that of Lemma 3.4, except that the relativehomotopy-liftings are replaced by local relative-homotopy-liftings.

Corollary 6.14. If $f: F \rightarrow G$ has the local RHLP with respect to the maps $\partial \Delta^{n} \hookrightarrow$ $\Delta^{n}$ for all $n \geq 0$ and $G$ is locally-fibrant, then $f$ also has the local RHLP with respect to all cofibrations $K \hookrightarrow L$ of finite simplicial sets. 
Proof. Every cofibration $K \hookrightarrow L$ can be constructed by a finite number of compositions and cobase changes from the generating cofibrations $\partial \Delta^{n} \hookrightarrow \Delta^{n}$.

Here is the main theorem of the paper:

\section{Theorem 6.15.}

(a) If $F$ and $G$ are locally-fibrant, then a map $F \rightarrow G$ is a local weak equivalence if and only if it has the local RHLP with respect to the maps $\partial \Delta^{n} \hookrightarrow \Delta^{n}$.

(b) If $F$ and $G$ are arbitrary and $\mathcal{R}$ is any fibrant-replacement functor for sSet, then a map $F \rightarrow G$ is a local weak equivalence if and only if $\mathcal{R} F \rightarrow \mathcal{R} G$ has the local RHLP with respect to the maps $\partial \Delta^{n} \hookrightarrow \Delta^{n}$.

Proof. For (a), we begin by assuming that $F$ and $G$ are locally-fibrant and that the map $f: F \rightarrow G$ has the local RHLP. By Corollary 6.14, it has the local RHLP with respect to all cofibrations between finite simplicial sets. In particular, it has the local RHLP with respect to $* \hookrightarrow S^{n}$; this proves condition (1) of Lemma 6.8 for $\pi_{n}^{l o c}(F, x) \rightarrow \pi_{n}^{l o c}(G, f x)$ (for $n=0$ one uses the RHLP with respect to $\emptyset \rightarrow *$ ). On the other hand, $f$ also has the local RHLP with respect to $S^{n} \vee S^{n} \hookrightarrow R H\left(S^{n}, *\right)$; this proves condition (2) of Lemma 6.8 for $\pi_{n}^{l o c}(F, x) \rightarrow \pi_{n}^{l o c}(G, f x)$. Here we use that local simplicial homotopy is an equivalence relation for locally-fibrant simplicial presheaves [J1, Lem. 1.9]. Now Lemma 6.7 and Proposition 6.9 tell us that we have a local weak equivalence.

We now assume that $f: F \rightarrow G$ is a local weak equivalence. To prove that it has the local RHLP with respect to the maps $\partial \Delta^{n} \hookrightarrow \Delta^{n}$ we follow exactly the same argument as in Section 5, observing that there are only finitely many applications of the various lifting properties.

To prove (b), let $\mathcal{R}$ be any fibrant-replacement functor for $s$ Set. We need only observe that $F \rightarrow G$ is a local weak equivalence if and only if $\mathcal{R} F \rightarrow \mathcal{R} G$ is one. Since $\mathcal{R} F$ and $\mathcal{R} G$ are objectwise-fibrant (hence locally-fibrant as well), part (a) applies.

\section{Applications}

Both the lifting characterization of local weak equivalences and the definition involving sheaves of homotopy groups are useful to have around. For instance, up to some technical difficulties in choosing basepoints, it is transparent from the homotopy group definition that the local weak equivalences have the two-out-ofthree property. This is awkward to show using the lifting characterization, however. We now give some results which are easy consequences of our lifting criterion.

Proposition 7.1 (Local right properness). Let $F \rightarrow G$ be a local weak equivalence between simplicial presheaves, and let $J \rightarrow G$ be a local-fibration. Then the map $J \times{ }_{G} F \rightarrow J$ is also a local weak equivalence.

Proof. The proof is the same as the proof of Corollary 4.2, except that liftings are replaced by local liftings. Note that $\mathrm{Ex}^{\infty}$ commutes with fibre-products of simplicial presheaves since both $\mathrm{Ex}^{\infty}$ and fibre-products are defined objectwise. Also, similar to observations in the proof of [J1, Prop. 1.17], $\mathrm{Ex}^{\infty}$ preserves local-fibrations.

Recall that a local acyclic fibration of simplicial presheaves is a map that is both a local weak equivalence and a local-fibration. The $(\Longrightarrow)$ direction of the following proposition was proved in $[\mathrm{J} 3$, Lemma 7$]$-we can now prove the other one (see [J3, Lemma 11] for a weaker version). 
Proposition 7.2. A map $p: F \rightarrow G$ of simplicial presheaves admits local liftings in every square

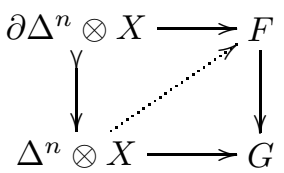

if and only if it is a local acyclic fibration.

Proof. First suppose that the local liftings exist. Then $p$ also has the local-lifting property with respect to the maps $\Lambda^{n, k} \hookrightarrow \Delta^{n}$, since these can be built from the maps $\partial \Delta^{r} \rightarrow \Delta^{r}$ by finitely many cobase-changes and compositions. Therefore $p$ is a local-fibration. Similar to observations in the proof of [J1, Prop. 1.17], the map $\operatorname{Ex}^{\infty} p: \operatorname{Ex}^{\infty} F \rightarrow \operatorname{Ex}^{\infty} G$ has the local lifting property with respect to all maps $\partial \Delta^{n} \otimes X \hookrightarrow \Delta^{n} \otimes X$. In particular, $\mathrm{Ex}^{\infty} p$ has the local RHLP with respect to the maps $\partial \Delta^{n} \hookrightarrow \Delta^{n}$; we use constant relative-homotopies. Using Theorem 6.15(b), $p$ is a local weak equivalence. This finishes one impliciation.

For the other direction, first assume that $F$ and $G$ are locally-fibrant. Since $F \rightarrow G$ is a local weak equivalence one gets local relative-homotopy-liftings by Theorem 6.15. Similar to the proof of Corollary 4.2, the fact that $F \rightarrow G$ is a local fibration allows one to homotope the local homotopy-liftings to get actual local liftings.

Now suppose that $p$ is an arbitrary local acyclic fibration, and suppose given a lifting square as in (7.3). As we have already observed, $\mathrm{Ex}^{\infty} p$ is also a local acyclic fibration, but with locally-fibrant domain and codomain. So by the previous paragraph there are local liftings for the composite square

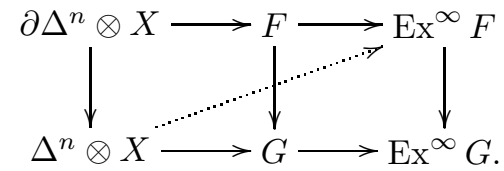

This translates to saying that for a sufficiently large $k$ there are local liftings in the square

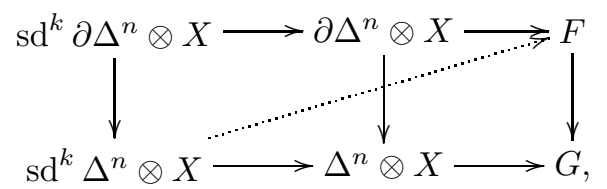

where the left horizontal maps are the 'last vertex maps' [GJ, p. 183].

Let $C$ be the mapping cylinder of $\operatorname{sd}^{k} \partial \Delta^{n} \rightarrow \partial \Delta^{n}$, and let $D$ be the mapping cylinder of $\operatorname{sd}^{k} \Delta^{n} \rightarrow \Delta^{n}$. Notice that $C$ is a subcomplex of $D$. Since the map $\mathrm{sd}^{k} \partial \Delta^{n} \otimes X \rightarrow F$ factors through $\partial \Delta^{n} \otimes X$, the constant homotopy ( $\mathrm{sd}^{k} \partial \Delta^{n} \times$ $\left.\Delta^{1}\right) \otimes X \rightarrow F$ factors through $C \otimes X$. Now, we have squares

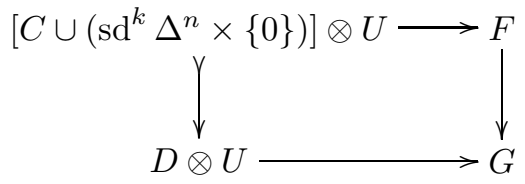


for all $U \rightarrow X$ in a covering sieve of $X$, where the maps $C \otimes U \rightarrow F$ and $D \otimes$ $U \rightarrow G$ are these 'constant homotopies'. The map $C \cup\left(\operatorname{sd}^{k} \Delta^{n} \times\{0\}\right) \rightarrow D$ is a trivial cofibration between finite simplicial sets (both the domain and codomain are contractible), so the square has a local lifting. By precomposing these liftings with the inclusion $\Delta^{n} \hookrightarrow D$, one obtains local liftings for the original square.

Corollary 7.4 (cf. [J3, Lemma 19]). Let $p: F \rightarrow G$ be a local-fibration, and let $i: K \hookrightarrow L$ be a cofibration of finite simplicial sets. If $p$ is a local weak equivalence or $i$ is a weak equivalence, then the induced map

$$
F^{L} \rightarrow F^{K} \times_{G^{K}} G^{L}
$$

is a local acyclic fibration.

Proof. To see that the map is a local acyclic fibration, it is enough by Proposition 7.2 to check that it has the local lifting property with respect to the maps $\partial \Delta^{n} \rightarrow \Delta^{n}$. By adjointness, one need only check that $F \rightarrow G$ has the local lifting property with respect to the map

$$
j:\left(L \times \partial \Delta^{n}\right) \cup\left(K \times \Delta^{n}\right) \rightarrow L \times \Delta^{n} .
$$

If $K \rightarrow L$ is an acyclic cofibration then so is $j$, and therefore the result follows from Lemma 2.2 and the definition of local fibration. If $F \rightarrow G$ was an acyclic fibration, then the result follows from Proposition 7.2 because $j$ is obtained by a finite number of cobase changes and compositions from the inclusions $\partial \Delta^{n} \rightarrow \Delta^{n}$.

Finally, we end with the following result. It is needed in [DHI], and we know of no proof that avoids the local lifting techniques we've just developed.

Corollary 7.5. Let $F \rightarrow G$ be a map between locally-fibrant simplicial presheaves, and let $K \hookrightarrow L$ be a cofibration of finite simplicial sets. If either map is a weak equivalence then the induced map $F^{L} \rightarrow F^{K} \times{ }_{G^{K}} G^{L}$ is a weak equivalence.

Proof. First, we know from [J1, Cor. 1.5] that both $F^{L}$ and $F^{K} \times_{G^{K}} G^{L}$ are locally-fibrant. A lifting square

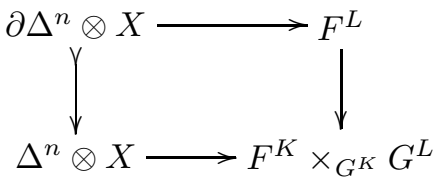

may be rewritten via adjointness as

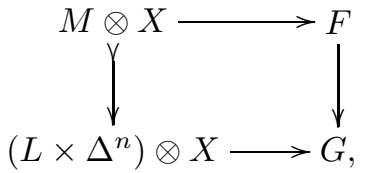

where $M=\left(L \times \partial \Delta^{n}\right) \cup\left(K \times \Delta^{n}\right)$. The map $M \hookrightarrow L \times \Delta^{n}$ is a cofibration between finite simplicial sets. When $F \rightarrow G$ is a local weak equivalence, Corollary 6.14 and Theorem 6.15(a) tell us that the above square has a relative-homotopy-lifting. Using adjointness once again, we get a relative-homotopy-lifting for the original square.

The other case is similar. If $K \hookrightarrow L$ is a weak equivalence then $M \hookrightarrow L \times \Delta^{n}$ is also one. So by the local version of Proposition 3.7 (which only works for acyclic 
cofibrations between finite simplicial sets), we have a relative-homotopy-lifting since $F$ and $G$ are locally-fibrant.

\section{REFERENCES}

[DHI] D. Dugger, S. Hollander, D. C. Isaksen, Hypercovers and simplicial presheaves, preprint, 2002.

[GJ] P. Goerss and J.F. Jardine, Simplicial homotopy theory, Progress in Mathematics 174, Birkhäuser, 1999.

[H] P. S. Hirschhorn, Localization of Model Categories, 2001 preprint. (Available at http://www-math.mit.edu/ psh).

[Ho] M. Hovey, Model Categories, Mathematical Surveys and Monographs vol. 63, Amer. Math. Soc., 1999.

[I] L. Illusie, Complexe cotangent et déformations I, Lecture Notes in Math. 239, Springer, 1971.

[J1] J.F. Jardine, Simplicial presheaves, J. Pure and Appl. Algebra 47 (1987), 35-87.

[J2] J.F. Jardine, Stable homotopy theory of simplicial presheaves, Canad. J. Math. 39 (1987), no. $3,733-747$.

[J3] J.F. Jardine, Boolean localization, in practice, Doc. Math. 1 (1996), 245-275.

[Jo] A. Joyal, unpublished letter to A. Grothendieck.

[K] D. M. Kan, On c.s.s. complexes, Amer. J. Math. 79 (1957), 449-476.

$[\mathrm{M}]$ J. P. May, Simplicial objects in algebraic topology, Van Nostrand Mathematical Studies 11 Van Nostrand, 1967.

[R] C. L. Reedy, Homotopy theory of model categories, preprint, 1974 (available online at http://www-math.mit.edu/ psh).

[Sp] E. H. Spanier, Algebraic Topology, Springer, 1966.

Department of Mathematics, Purdue University, West Lafayette, IN 47907

Department of Mathematics, University of Notre Dame, Notre Dame, IN 46556

E-mail address: ddugger@math.purdue.edu

E-mail address: isaksen.1@nd.edu 